\title{
An automated ranking platform for machine learning regression models for meat spoilage prediction using multi-spectral imaging and metabolic profiling
}

\author{
Lucia Estelles-Lopez ${ }^{a}$, Athina Ropodi ${ }^{b}$, Dimitris Pavlidis ${ }^{b}$, Jenny Fotopouloub, \\ Christina Gkousari $^{b}$ Audrey Peyrodie ${ }^{a}$, Efstathios Panagou ${ }^{b}$, George-John Nychas $^{b}$, \\ Fady Mohareba, ${ }^{*}$
}

a Bioinformatics Group, Department of Agrifood, School of Water, Energy and Environment Cranfield University, College Road, Cranfield, Bedfordshire, MK43 OAL, UK bLaboratory of Microbiology and Biotechnology of Foods, Department of Food Science and Technology, Agricultural University of Athens, lera Odos 75, Athens, Greece, GR-11855

Bioinformatics Group, Department of Agrifood, School of Water, Energy and Environment, Cranfield University, College Road, Cranfield, Bedfordshire, MK43 OAL, UK, Tel: +44 (0) 7775884779. Email address: f.mohareb@cranfield.ac.uk

Keywords: Machine Learning - Pattern Recognition - Meat spoilage - Metabolic profiling Data science - Food Quality.

\author{
Abbreviations: \\ SVM-R: Support Vector machines regression \\ RF-R: Random forests regressions \\ kNN: k-nearest neighbour \\ PCA: Principal Component Analysis \\ OLS-R Ordinary least squares regression \\ SL-R: Stepwise Linear regression \\ $M L$ : Machine Learning \\ RMSE: Root mean square of error \\ PC-R Principal Component regression
}




\section{Abstract}

Over the past decade, analytical approaches based on vibrational spectroscopy, hyperspectral / multispectral imagining and biomimetic sensors started gaining popularity as rapid and efficient methods for assessing food quality, safety and authentication; as a sensible alternative to the expensive and time-consuming conventional microbiological techniques. Due to the multi-dimensional nature of the data generated from such analyses, the output needs to be coupled with a suitable statistical approach or machine-learning algorithms before the results can be interpreted. Choosing the optimum pattern recognition or machine learning approach for a given analytical platform is often challenging and involves a comparative analysis between various algorithms in order to achieve the best possible prediction accuracy.

In this work, "MeatReg", a web-based application is presented, able to automate the procedure of identifying the best machine learning method for comparing data from several analytical techniques, to predict the counts of microorganisms responsible of meat spoilage regardless of the packaging system applied. In particularly up to 7 regression methods were applied and these are ordinary least squares regression, stepwise linear regression, partial least square regression, principal component regression, support vector regression, random forest and $k$-nearest neighbours.

MeatReg" was tested with minced beef samples stored under aerobic and modified atmosphere packaging and analysed with electronic nose, HPLC, FT-IR, GC-MS and Multispectral imaging instrument- Population of total viable count, lactic acid bacteria, pseudomonads, Enterobacteriaceae and B. thermosphacta, were predicted. As a result, recommendations of which analytical platforms are suitable to predict each type of bacteria and which machine learning methods to use in each case were obtained. The developed system is accessible via the link: http://elvis.misc.cranfield.ac.uk/SORF/. 


\section{Introduction}

Consumers demand food products, which should not be only perfectly safe for human consumption, but visually attractive as well. Additionally, to be more likely to meet the customers' expectations, foodstuff should include fewer additives, be minimally processed and high in quality. (Van Wezemael, Verbeke, de Barcellos, Scholderer, \& Perez-Cueto, 2010). In general, food products are considered spoiled if it is unacceptable by the customer, though not necessarily unsafe (Koutsoumanis, 2009). This is also the case with fresh meat, where spoilage is quite a subjective judgement among consumers, often based in the presence of gross discoloration, strong off-odours and development of slime due to intrinsic or extrinsic factors (G. J. Nychas, P. N. Skandamis, C. C. Tassou, \& K. P. Koutsoumanis, 2008).

Meat spoilage is a very complex phenomenon, which involves significant changes and activities of very different microbial groups depending on the storage conditions e.g. packaging, temperature (Doulgeraki, Ercolini, Villani, \& Nychas, 2012). However, according to EU authorities (Commission, 2005), the quality of fresh meat is evaluated only by viable counts of bacteria able to grow on very generic medium (Total viable count) or on counts of the Enterobacteriaceae family. On the other hand, it is well established that pseudomonads are the major cause of spoilage in aerobic conditions as once they have used all glucose and lactate available, they start to metabolise the nitrogen sources, producing slime and off-odours (Mohareb et al., 2015). Under modified atmosphere packaging, other microorganisms like $B$. thermosphacta, Enterobacteriaceae and lactic acid bacteria are dominating, whilst under vacuum packaging the dominant species are Pseudomonas spp., B. thermosphacta and S. putrefaciens (G-J.E; Nychas, Marshall, \& Sofos, 2007). Counting colonies is certainly time-consuming, costly and provide retrospective information (G. J. E. Nychas, P. N. Skandamis, C. C. Tassou, \& K. P. Koutsoumanis, 2008). Moreover, both the analysis of limited samples and/or their low counts, can significantly underestimate the microbial contribution to meat quality. Recently, rapid, non-invasive methods relying on processing large datasets using computational analysis are gaining popularity (George-John E. Nychas, Panagou, \& Mohareb, 2016). Although such instruments represent an efficient alternative to conventional microbiological analysis, the experimental output is far more complex and usually needs processing before the results can be interpreted. In the food sector, a plethora of machine learning approaches has been followed by different authors in order to predict spoilage in meat samples using metabolomics data (Comprehensive examples are highlighted in (Ropodi, Panagou, \& Nychas, 2016). internet technologies and more specifically open sources platforms will enhance food safety management system (George-John E. Nychas et al., 2016) while will allow supply chains to use virtualizations dynamically in operational management processes. This will improve support for food companies dealing with perishable products, unpredictable supply variations and stringent food 
safety and sustainability requirements.

While research involving metabolomics data in tandem with machine learning techniques is extensive, guidelines to choose the machine learning method that provides the best results for a specific type of data are still needed. Furthermore, the actual procedure of optimising and validating the spoilage prediction models is computationally extensive, and often requires the availability of suitable resources and statistical knowledge.

Therefore, the aim of this work is (i) to develop spoilage prediction models from data derived from different analytical instruments, and (ii) to implement an accuracy ranking system through a platform (MeatReg), which assesses the suitability of machine learning methods to specific type of metabolic data provided by a certain analytical process. For this study, metabolomics data from minced beef samples stored under aerobic and modified atmosphere packaging were collected using 5 different analytical and imaging instruments: electronic nose (eNose), High Performance Liquid Chromatography (HPLC), Fourier Transformed Infrared Spectroscopy (FT-IR), Gas Chromatography coupled to Mass Spectrometry (GC-MS) and multi-spectral imaging (MSI).

In particular in this study, seven machine learning methods; namely Ordinary Least Squares regression (OLS-R), Stepwise Linear regression (SL-R), Principal Component regression (PC-R), Partial Least Squares Regression (PLS-R), Support Vector Regression (SVM-R), Random Forests Regression (RF-R) and k-Nearest Neighbours' Regression (kNN-R) were used to predict bacterial counts for Pseudomonads, Lactobacilli, B. thermosphacta and Enterobacteriaceae, as well as for the bacterial total viable count. This way, the most suitable analytical platforms to predict bacterial counts for each type of bacteria present in meat stored under aerobic or modified atmosphere conditions were identified and machine-learning methods were ranked for each scenario according to their performance. Additionally, "MeatReg" was made available online as a web-based application in order to provide a flexible and setup-free mean to automate the whole analysis process since internet technologies and more specifically open sources platforms will enhance food safety management system (George-John E. Nychas et al., 2016) while will allow supply chains to use virtualizations dynamically in operational management processes. This will improve support for food companies dealing with perishable products, unpredictable supply variations and stringent food safety and sustainability requirements.

\section{Material and methods}

\subsection{Sample preparation $\&$ microbiological analyses}


Fresh minced meat was obtained from a central butcher shop in Athens and transported under refrigeration to the laboratory within $30 \mathrm{~min}$. Portions of approximately $75-80 \mathrm{~g}$ were placed on styrofoam trays, were stored in air or under modified air packaging (MAP) conditions $\left(20 \% \mathrm{CO}_{2} /\right.$ $80 \% \mathrm{O}_{2}$ ) at 4 and $10^{\circ} \mathrm{C}$. For aerobic storage, samples were covered with plastic food membrane for domestic use and for MAP storage, samples were packed into plastic pouches of gas permeability at $20^{\circ} \mathrm{C}$ and $50 \%$ relative humidity of ca. 25 and $90 \mathrm{~cm}^{3} / \mathrm{m}^{2}$ per day $/ 10^{5} \mathrm{~Pa}$ for $\mathrm{CO}_{2}$ and $\mathrm{O}_{2}$ respectively, using a HenkoVac 1900 Machine. At appropriate time intervals (approximately every 24 and 12 hours for the case of $4^{\circ} \mathrm{C}$ and $10^{\circ} \mathrm{C}$ respectively), multispectral images of duplicate samples were captured and samples were analyzed microbiologically until spoilage was pronounced and sub-samples were stored $\left(-20^{\circ} \mathrm{C}\right)$ for FTIR, HPLC, GC-MS and e-nose measurements. Additionally, three more samples at 0 hours (control samples) were analyzed. In total, 105 samples (three control samples and 11-14 duplicate samplings per packaging condition per storage temperature) were analyzed. Generally speaking, meat spoilage and sample discoloration was not evident to the naked eye, except between extreme storage times (See Supplementary Figure S4). Twenty-five gram-portions from each meat samples were weighted aseptically in $400 \mathrm{ml}$ sterile stomacher bags (Seward Medical, London, United Kingdom), containing $225 \mathrm{ml}$ of sterile quarter Ringer's solution (LabM Limited, Lancashire, United Kingdom) and were homogenized for $60 \mathrm{sec}$ (Lab Blender 400, Seward Medical). Appropriate serial dilutions were prepared with the same Ringer's solution and duplicate 0.1 or $1 \mathrm{~mL}$ samples of the appropriate dilutionswere spread or mixed on the following media: plate count agar (PCA, Biolife 4021452, Milano, Italy) for total viable counts (TVC), incubated at $30^{\circ} \mathrm{C}$ for $48-72 \mathrm{~h}$; Pseudomonas agar base (PAB, Biolife 401961,Milano, Italy) for Pseudomonas spp., incubated at $25^{\circ} \mathrm{C}$ for $48-72$ h;streptomycin thallous acetate-actidione agar (STAA, Biolife 402079, Milano, Italy) for $B$. thermosphacta, incubated at $25^{\circ} \mathrm{C}$ for $72 \mathrm{~h}$; and de Man-Rogosa-Sharpe medium (MRS, Biolife, 4017282, Milano, Italy) with $\mathrm{pH}$ adjusted to 5.7 with $10 \mathrm{~N} \mathrm{HCl}$, for lactic acid bacteria overlaid with the same medium and incubated at $30^{\circ} \mathrm{C}$ for $48-72 \mathrm{~h}$. All plates were examined visually for typical colony types and morphological characteristics that were associated with each growth medium. Moreover, the selectivity of each medium was routinely checked by Gram staining and microscopic examination of smears prepared from randomly selected colonies obtained from the media.

\subsection{Fourier Transform Infrared (FTIR) Spectroscopy}

FTIR spectra were acquired using an FT/IR 6200 JASCO spectrometer (Jasco Corp., Tokyo, Japan). Small portions ( $3 \mathrm{~g}$ ) were placed on the surface of a $\mathrm{ZnSe} 45^{\circ}$ HATR (Horizontal Attenuated Total Reflectance) crystal (PIKE Technologies, Madison, Wisconsin, United States) and, using the Spectra Manager software version 2 (Jasco Corp.), spectra were collected from 4000 to $400 \mathrm{~cm}^{-1}$ (100 scans, resolution of $4 \mathrm{~cm}^{-1}$ ) within a period of $2 \mathrm{~min}$. Prior to the measurements, reference spectra were acquired using the crystal with no added meat. After each measurement, 
the crystal's surface was cleaned using firstly detergent and distilled water and secondly with analytical grade acetone, and dried with lint-free tissue.

\subsection{Image Acquisitions and Analysis}

Images from every sample were captured using Multispectral imaging (MSI), a system which acquires multispectral images in 18-non-uniformly distributed wavelengths ranging from 405 to 970nm in visible and short-NIR spectral region. The system's detailed description commercialized by "Videometer A/S (VM)" (http://www.videometer.com) has been reported elsewhere (Panagou, Papadopoulou, Carstensen, \& Nychas, 2014). The system was calibrated radiometrically and geometrically using well-defined standard targets, after a light setup based on the type of object to be recorded called "autolight". Petri dishes (75-80 g meat portions) were placed inside an Ulbricht sphere in which the camera is top-mounted. An image-processing step is needed that will result in an image mask where only meat tissue is included. This step was implemented using VideometerLab software (version 2.12.39). The resulting image naturally includes irrelevant information such as the Petri dish and its surrounding background, as well as the fat and connective tissue within the meat. For this reason, an image pre-processing step was performed; which includes transformation and segmentation procedures using the default Videometer lab software which controls the operation of the instrument. This procedure is described in details elsewhere (Ropodi, Pavlidis, Mohareb, Panagou, \& Nychas, 2015). Briefly, Canonical discriminant analysis (CDA) was applied to separate the images according to the regions of interest (Daugaard, AdlerNissen, \& Carstensen, 2010). Following this transformation, the separation was distinct, and a simple threshold was enough to separate meat from non-meat pixels. The resulting segmented image for each sample only contains the isolated part of the meat tissue as the main region of interest (ROI) was used for the extraction of spectral data used for the statistical modelling. For this step, the mean reflectance spectrum was calculated for each image by averaging the intensity of pixels within the ROI at each wavelength. Furthermore, the standard deviation of the pixels' intensity per wavelength was extracted. The resulting data consisted of 18 mean values and 18 standard deviations of the reflectance, as it was recorded by the camera for the pixels that were included in each image's ROI, and were further analyzed with various mathematical modelling methods.

\subsection{Electronic nose}

The volatile compounds chemical fingerprints of beef fillet samples were analysed using a gas sensor array system (LibraNose, Technobiochip, Napoli, Italy). The platform uses an array of 8 quartz crystal microbalance (QMB) non-selective sensors coated with different poly-pyrrole derivatives, synthesized at Technobiochip. Further details on the LibraNose instrumentation and mode of action can be found elsewhere (Baietto, Wilson, Bassi, \& Ferrini, 2010). For each measurement, a beef fillet sample of $5 \mathrm{~g}$ was introduced inside a $100 \mathrm{ml}$ volume glass jar and left at 
room temperature $\left(20^{\circ} \mathrm{C} \pm 2^{\circ} \mathrm{C}\right)$ for $15 \mathrm{~min}$ to enhance desorption of volatile compounds from the meat into the headspace. The headspace was then pumped over the sensors of the electronic nose and the generated signal was continuously and in real time recorded and stored to a laptop computer.

\subsection{High Performance Liquid Chromatography (HPLC)}

The analysis was performed as described by (Skandamis \& Nychas, 2001) equipped with a Model PU-980 Intelligent pump, a Model LG-980-02 ternary gradient unit pump and a MD-910 multi wavelength detector. The injection valve was connected with a $20 \mu \mathrm{L}$ loop, whilst $50 \mu \mathrm{L}$ of the sample was injected each time. The sample was eluted isocratically with a solution of $0.009 \mathrm{~N}$ $\mathrm{H}_{2} \mathrm{SO}_{4}$ (using HPLC grade solvent and ultra-pure water) through an Amminex HPX-87H column (300 × $7.8 \mathrm{~mm}$, Bio-Rad Laboratories, Richmond, CA, USA) at a rate of $0.7 \mathrm{~mL} / \mathrm{min}$ and oven temperature set at $65^{\circ} \mathrm{C}$. The software used for the collection and the processing of the spectra was the Jasco Chrompass Chromatography Data system v1.7.403.1. Spectral data were collected from 200 to $600 \mathrm{~nm}$, however chromatogram integration was performed at $210 \mathrm{~nm}$ and the purity of the peaks was examined through the software using all spectral ranges. Solutions of oxalic, citric, malic, lactic, acetic, formic, tartaric, succinic and propionic acids (HPLC grade) were used as reference substances, analysed using the same programme and their spectra were compared with the samples for the identification of the peaks.

\subsection{Gas Chromatography coupled to Mass Spectrometry (GC-MS)}

GC-MS analyses was performed as described by (A. A. Argyri, Mallouchos, Panagou, \& Nychas, 2015). Briefly, Agilent 7890A gas chromatograph coupled to Agilent $5973 \mathrm{C}$ mass spectrometer was deployed using helium as a carrier gas at a constant flow rate of $1 \mathrm{~mL} / \mathrm{min}$. The injection port was equipped with a liner $(0.75 \mathrm{~mm}$ i.d.) suitable for SPME analysis. It was operated in splitless mode for $1 \mathrm{~min}$ at $250{ }^{\circ} \mathrm{C}$. Separation of compounds was performed on an HP-5MS column $(30 \mathrm{~m}, 0.25 \mathrm{~mm}$ i.d., $0.25 \mu \mathrm{m}$ film thickness, Agilent). Oven temperature was maintained at $40{ }^{\circ} \mathrm{C}$ for $5 \mathrm{~min}$, programmed at $4{ }^{\circ} \mathrm{C} / \mathrm{min}$ to $150{ }^{\circ} \mathrm{C}$ and then it was raised to $250{ }^{\circ} \mathrm{C}$ with a rate of $30{ }^{\circ} \mathrm{C} / \mathrm{min}$ and held for $5 \mathrm{~min}$. The interface temperature was set at $280^{\circ} \mathrm{C}$. The mass spectrometer was operated in electron impact mode with the electron energy set at $70 \mathrm{eV}$ and a scan range of 29-350 m/z (scan rate: 4.37 scans/s, gain factor: 1 , resulting EM voltage: $1188 \mathrm{~V}$ ). The temperature of MS source and quadrupole was set at 230 and $150{ }^{\circ} \mathrm{C}$, respectively. Identification of the compounds was conducted as described elsewhere (A. A. Argyri et al., 2015).

\subsection{Mathematical modelling}

Three sets of minced beef samples stored under different packaging conditions; aerobic packaging 
(AIR), modified atmosphere packaging (MAP) and mixed packaging storage (AIR + MAP) were analysed using different analytical and imaging platforms as shown is Figure 1, where output datasets were used to implement and test the performance implemented with "MeatReg". For each of the sets, data from 5 analytical platforms used in metabolomics in order to predict bacterial counts grown in 5 different mediums (a total of 50 combinations; 2 packaging systems $x 5$ platform $\mathrm{x}$ 5 species counts) was analysed. The analysis was performed using the open-source software environment $R$ as it contains several resources and support to perform machine learning. For each dataset corresponding an analytical platform, a Principal Component Analysis (PCA) plot was generated for exploratory purposes. The models were generated using seven different methods for machine learning as previously outlined. In order to generate each of the models, the dataset was randomly split over 20 iterations into a training dataset, which contained $70 \%$ of the samples, and a testing dataset composed by the remaining samples. The training dataset was then used to build the model while the testing dataset was used to calculate the performance of such model.

Ordinary least squares regression (Wilkinson \& Rogers, 1973), OLS-R, and stepwise linear regression (Friedman, Hastie, \& Tibshirani, 2010), SL-R, are linear regression methods, with the difference that linear stepwise linear regression performs feature selection. These were performed using the "Im" function present in the package "stats" in R base. Principal component regression (Kendall, 1957), PC-R, and partial least squares regression (Höskuldsson, 1988), PLS-R, perform the regression models based on the reduction the number of variables to a few components that explain the variability within the samples. These methods were implemented using the "pcr" and "pls" functions with 3 components from the "pls" package (Mevik \& Wehrens, 2007).

Random forest (Breiman, 2001) is a method based on ensemble learning that combines the predictions of several decision trees in order to provide a more accurate prediction than the individual trees on their own. It was implemented with 200 trees using the "randomForest" function from the "randomForest" package (Liaw \& Wiener, 2002). For Support Vector Machines Regression (Boser, Guyon, \& Vapnik, 1992), SVM-R, an optimal hyperplane that minimizes the distance to all the data points is built. As it is a parametric method, 3 parameters, cost of constraints violation, epsilon and gamma, were adjusted in order to optimize the model. To find the best values for these parameters grid search was carried out. The implementation was performed using the "svm" function from the "e1071" package (Dimitriadou, Hornik, Leisch, Meyer, \& Weingessel, 2011) and the kernel selected was the radial basis kernel (RBF). The values for grid search were $0,0.03,0.06$, $0.09,0.20,0.30,0.60$ and 0.90 for gamma; $0,0.02,0.04,0.06,0.10,0.20,0.30$ and 0.60 for epsilon; and $1,4,7,10,13,16,19,30,50,70,90$ and 110 for the cost. Finally, $k$-nearest neighbours (Silverman \& Jones, 1989), kNN, predicts the value of an unknown sample based on the values of the $k$ samples closer in distance. It was implemented using the function "knn.reg" from the "FNN" package (Beygelzimer, Kakadet, Langford, Arya, \& Mount, 2013). The best $k$ was selected using 
grid search from $\mathrm{k}=4$ to 10 .

For the methods that required grid search the dataset was split into training and testing. The training dataset was used to perform a model for each one of the possibilities of the grid search. The performance of each one of the models was calculated using the testing dataset and the parameters, which provided the model with the lowest RMSE, were selected.

\subsubsection{Models validation}

The performance of the models was assessed in terms of how close the predicted values were to the observed. Quantification was performed by calculating the root-mean-square error (RMSE), the accuracy, the maximum difference between predicted and observed values $\left(\Delta_{\max }\right)$, the bias factor $\left(B_{f}\right)$ and the accuracy $\left(A_{f}\right)$ factor (Baranyi, Pin, \& Ross, 1999). The root mean-square error (Equation 1) quantifies the difference between predicted and observed value. If the difference is small, the RMSE is a positive number close to 0 .

$$
R M S E=\sqrt{\frac{\sum(\text { predicted }- \text { observed })^{2}}{n}}
$$

\section{Equation 1. Root mean-square error formula.}

The accuracy factor (Equation 2) is an indicator of the difference between observed and predicted values. The bias factor (Equation 3 ) indicates if there is systematic bias, providing information about how much the model over-predicts or under-predicts. Both factors are close to 1 if the model provides good predictions.

$$
A_{f}=10\left(\frac{\sum \log \left(\left|\frac{\text { predicted }}{\text { observed }}\right|\right)}{n}\right)
$$

Equation 2. Accuracy factor formula.

$$
B_{f}=10\left(\frac{\sum \log \left(\frac{\text { predicted }}{\text { observed }}\right)}{n}\right)
$$

\section{Equation 3. Bias factor formula.}

The accuracy is the percentage of samples correctly predicted (Equation 4). A sample is considered correctly predicted if the difference between predicted and observed values is less than $1 \log$ (TVC) (Mohareb, Papadopoulou, Panagou, Nychas, \& Bessant, 2016) as it is the equivalent to 1 dilution. 


$$
\text { Accuracy }=\frac{\text { samples correctly predicted }}{\text { overall number of samples }} \times 100
$$

\section{Equation 4. Accuracy formula.}

The maximum difference between predicted and observed values was calculated as well. Even if it is not a statistical parameter, it is informative as it shows the worst prediction. If the highest difference between predicted and observed is below or close to 1, the model is providing good predictions but if it is higher it means that the worst prediction deviates too much from the observed values.

Validation is a crucial part of the analysis. To assess the performance of the machine learning algorithms, Monte Carlo cross validation (Qing-Song Xu \& Liang, 2001) was used. For $K=20$, the samples from each dataset were randomly distributed into training and testing datasets in 20 different splits. Then, the performance is calculated as an average of the performance of the 20 models. Performance plots for each scenario and machine learning method applied were generated to observe how the calculations of accuracy vary depending on the value of $\mathrm{N}$.

\subsubsection{Ranking and visualization}

Machine learning methods were ranked according to the RMSE for each scenario. The first machine learning method in the ranking were used to generate a summary plot in which the intersection of analytical platform and the medium used to grow microorganisms indicates the performance of the best machine learning technique (Figure 2). Light green tones represent that at least one of the machine learning methods applied provided a good prediction of the bacterial count whilst red tones illustrate that no machine learning methods provided a good performance.

\section{Results}

\subsection{Microbial Association}

The microbial association of minced meat comprises of Total Viable Counts (initial counts; 5.65 log cfu/g), Pseudomonads (4.13 log cfu/g), Brochothrix thermosphacta (4.24 log cfu/g), Lactic acid bacteria (4.33 log cfu/g), Enterobacteriaceae (1.92 log cfu/g) and yeasts (4.80 log cfu/g). Tables 1 and 2 summarize kinetic parameters of the member of microbial association following data fitting using the Baranyi and Roberts' model (Baranyi \& Roberts, 1994). Lag phase was observed only at $0^{\circ} \mathrm{C}$, while there was an increase in the maximum specific growth rate $\left(\mu_{\max }\right)$ of various groups correlated to the increase of the storage temperature regardless the packaging type. The influence of the packaging type was also evident. For example, TVC $\mu_{\max }$ followed the increase order MAP < 
AIR, regardless the storage temperature.

\subsection{Performance of Machine-learning models in data acquired from different metabolomics fingerprint profiles}

Ranking of the tested machine learning models for AIR and MAP, and AIR+MAP is provided in the supplemented Table S1, S2 and S3 respectively; while the best performed models are indicated in Figures 2. It should be noted that no data pre-treatment was performed prior to analysis. Taking RMSE as a measure of model's performance validation, the data analysis revealed that these values ranged from 0.370 to 1.321 across different best machine learning methods to predict each of the species counts for AIR, MAP, conditions (Figures 2a \& 2b). Furthermore, for mixed storage conditions (AIR + MAP) showed comparable results to storage-specific models; with RMSE values ranged from 0.388 to 1.343 (Figure 2c).

Under AIR packaging storage (Figure 2a), the best overall prediction outcomes were obtained from data derived with HPLC and MSI. HPLC achieved $100 \%$ prediction accuracy for Lactobacilli (medium MRS, RMSE = 0.466) when combined with RF-R, 93.9\% and $92.6 \%$ when combined with kNN-R for total viable count (medium PCA, RMSE $=0.508$ ) and B. thermosphacta (medium STAA, RMSE $=0.564$ ) respectively. eNose achieved $86.6 \%$ for Enterobacteriaceae (medium VRBG, RMSE $=0.724$ ). Pseudomonads (medium CFC), were best predicted using MSI combined with PLS-R (RMSE = 0.853). The ranking of the entire machine learning methods suite applied to beef stored under aerobic packaging conditions for each combination is available in the Supplementary Materials - Table $1 \mathrm{~S}$.

For MAP storage (Figure $2 b$ ), the RMSE ranged from 0.370 to 0.866 across different machine learning methods to predict each of the species counts. RF-R provided the best predictions in most cases. For electronic nose, the RMSE ranged from 0.431 for Lactobacilli (medium MRS) to 0.654 for Pseudomonads. For FT-IR, the RMSE ranged from 0.574 for Lactobacilli to 0.866 for Enterobacteriaceae. For GC-MS data, the RMSE ranged from 0.426 for Lactobacilli to 0.621 for $B$. Thermosphacta and the accuracy is above $89 \%$ for all bacterial counts. On the other hand, $k N N-R$ and RF-R provided the best results for HPLC and MSI. For HPLC data, the RMSE ranged from 0.378 and $100 \%$ accuracy using RF-R for Lactobacilli to 0.668 and $91.5 \%$ accuracy using kNN-R for Enterobacteriaceae. For MSI measurements, the RMSE ranged from 0.506 and $94.1 \%$ accuracy for Lactobacilli, to 0.822 and $73.0 \%$ accuracy for $B$. thermosphacta (medium STAA), both using RF-R. The best predictions for Enterobacteriaceae, were obtained using GC-MS, with an RMSE of 0.480 and an accuracy of $93.9 \%$. For Lactobacilli (medium MRS) the highest RMSE was obtained using HPLC in tandem with RF-R. For the total viable counts, GC-Ms and eNose in tandem with SV-R and 
RF-R provided predictions with an RMSE of 0.561 and 0.564 respectively. The best predictions for pseudomonads, were obtained using GC-MS with $k N N-R$, with an RMSE of 0.471 and $96.0 \%$ accuracy. Finally, the best predictions for $B$. thermosphacta, was obtained using eNose combined with RF-R; achieving RMSE of 0.566 and an accuracy of $92.8 \%$. The ranking of the entire machine learning methods applied to beef stored under modified atmosphere packaging conditions for each combination is available in the Supplementary Materials - Table S2.

In the case of mixed packaging system, the input dataset consisted of a random mixture of AIR and MAP within a single dataset and the packaging type was not included as a model input variable, in order to evaluate the performance of the developed pipeline in achieving a good prediction accuracy regardless of the packaging system applied. For this experiment, GCMS coupled with RF-R achieved the best prediction accuracy throughout all growth media, followed by HPLC coupled with $\mathrm{KNN}-\mathrm{R}$, while data derived from FT-IR had the worst prediction performance (Figure 2c). The best predictions for Enterobactriaceae (VRBG), B, thermosphacta (STAA), Lactobacilli (MRS), Pseudomonads (CFC) and total viable counts (PCA), were all achieved using random forest at an RMSE at $0.558,0.568,0.368,0.66$, and 0.471 respectively. The ranking of the entire machine learning methods applied to beef stored under mixed packaging conditions for each combination is available in the Supplementary Materials - Table S3.

\subsection{Automation of the ML ranking system via an interactive web-based platform}

In order to maximise benefit to the food research community from the developed protocol, the entire process of models development, optimisation and validation pipeline has been deployed within an tailored R library package,"MeatReg” deployed within a Web application. The purpose of this package is to automate the procedure of creating prediction models based on data from analytical platforms and microbiological counts in CSV format. Figure 3 shows the general workflow of the analysis. The program loops through machine learning methods, datasets from analytical platforms and microbial data selected (highlighted in red) in order to calculate the performance of the models for all possible combinations. For each dataset from an analytical platform, a Principle Component analysis (PCA) plot is provided (highlighted in blue). For each combination of machine learning method, data from the analytical platform and microbial counts are combined and divided into training and testing datasets, models are created and statistical parameters are calculated $\mathrm{K}$ times and averaged (highlighted in green).

The "MeatReg" package is available to use via the sorfML web application platform 
(http://elvis.cranfield.ac.uk/SORF/), allowing users to perform regression analysis online (Figure 4). For a specific dataset already uploaded into the sorfML database, users select "Regression analysis" and they are able to select the analytical platforms, bacterial growth mediums and machine learning methods to include in their analysis. Additionally, other parameters can be modified, such as select a pre-treatment, convert bacterial counts to logarithmic scale if they were not already converted, modify the number of trees for random forest or modify the parameters for grid search for kNN-R and SVM-R. When the user clicks "Submit" it passes all the selected options to a Python script as command line arguments. A Python script generates and executes an $\mathrm{R}$ script to perform the regression analysis using the "MeatReg" package, gathers the information into a LaTeX file and it is presented to the user as a PDF report containing the summary plot, the ranking for machine learning methods, performance plots, PCA plots and the R code to run the analysis.

\section{Discussion}

Data mining derived from food analyses using rapid analytical techniques based on limited or nondestructive / non-invasive sensors is a growing area in the food sector (George-John E. Nychas et al., 2016). Indeed, the implementation of these rapid techniques coupled with data analysis methods has given promising results in several food products (Ropodi et al., 2016). To our knowledge, "MeatReg" is the first attempt to compare machine-learning methods to determine the suitability of several analytical instruments in predicting microbiological quality, including spoilage of foods and more specifically meat, once a suitable dataset is uploaded to the platform. With "MeatReg", it is possible to visualize how data derived from different analytical instruments are suitable to predict not only the general microbiological quality of meat but also determine the contribution of individual bacterial species in the food spoilage process, using a combination of seven regression methods. All the machine-learning methods applied are ranked according to their performance, in order to provide information about how each of them performed individually, and in relation to one another. This multi 'dimensional' analyses could be used to evaluate (i) spoilage, regardless the storage conditions e.g. temperature, packaging etc, and (ii) the feasibility of using in food sector specific non-destructive/non-invasive instruments/sensors.

For example, Pseudomonads and Enterobacteriaceae counts are well known to be related with meat spoilage under aerobic packaging conditions (Nychas et al. 2008). In this case the most suitable sensor is MSI (Figure 2) and it is quite convenient due to its portability, i.e. it can be placed on line within the food industry production line, and it is able to provide very fast and accurate results. On the other hand, although HPLC and GC-MS usually provide accurate prediction, they are not portable and cannot be deployed within the production chain, however instead can be used by competent authorities in their analytical laboratories and to conclude regarding the 
microbiological quality as requested by EFSA's legislation (EFSA 2005). Electronic nose and FT-IR are also convenient methods and although do provide good prediction $>86 \%$ for TVC, the lack of accuracy in the case of pseudomonads in meat stored under aerobic packaging, can be considered as a drawback if these organisms is requested to be used as quality index. In general, this pipeline helps to decide which analytical technique to be used by whom (e.g. meat industry, food authority) and where (on in at line) to predict all types of bacteria of interest according to the food product being tested, and which machine learning algorithms will provide accurate predictions of their bacterial counts without having to invest in numerous and usually expensive platforms.

Models developed using "MeatReg" are validated using the Monte Carlo cross validation as opposed to the more widely applied methods such as leave-one-out cross-validation (LOOCV). Though LOOCV is a very popular validation method (Argyri et al., 2010; Mohareb et al., 2016; Wang, Wang, Liu, \& Liu, 2012), it has been shown that using Monte Carlo validation can avoid an unnecessary large model and decrease the risk of overfitting (Qing-Song Xu, Liang, \& Du, 2004). Other common strategy is to randomly split the original dataset into training and testing datasets (Anthoula A. Argyri et al., 2013; Mataragas, Skandamis, Nychas, \& Drosinos, 2007; Panagou, Mohareb, Argyri, Bessant, \& Nychas, 2011; Rajamäki et al., 2006), but the calculation of the statistical parameters highly depends on which samples fell into each dataset. However, with Monte-Carlo validation the original datasets are split into training and testing datasets $\mathrm{K}$ number of times (as specified by the user). Training datasets are used to build the statistical model and testing datasets are used to test it. $\mathrm{K}$ models are built and tested and the statistical parameters for all of them are averaged. In contrast to splitting the dataset into training and testing datasets only once, this method is more reliable to assess the performance as it does not depend that much in which samples randomly fall into the training and testing subsets (Mohareb et al., 2016). Moreover, a particular effort has been placed in providing a comprehensive and easy to understand report. $A$ heatmap that ranks all included instruments/sensors, algorithms and species counts using a redgreen colour-ramp; where red represents unsuitability and green represents suitability (Figure 2). In this way, it became possible to compare and rank several possible scenarios within a single plot. Detailed information about each scenario is also provided within the generated report. For each combination of analytical platform and bacteria, all the tested machine-learning methods are ranked and statistics are provided for all of them. Performance plots are shown as well. Principal Component Analysis (PCA) plots are provided hereafter for all the data from analytical platform. PCA is a very popular unsupervised method for exploratory purposes and it is worth checking the plots for the experimental data in order to detect if it behaves as expected or to find possible outliers as well as giving insights about the potential for a given dataset to be used to optimise an accurate model; through the identification of correlation between samples . Finally, the R code applied is shown in the report as well in order to keep track of the selected parameters. 
As far as the models performance for each of the applied analytical instruments is concerned, MSI, HPLC and GC-MS provide good predictions for all types of counts. The predictions regarding Enterobacteriaceae and $B$. thermosphacta are limited for FT-IR and electronic nose respectively while both failed to predict pseudomonads counts under aerobic packaging. Although most of the research has been performed using PLS-R or support vector machines, our results show that other machine learning methods such as kNN-R and RF-R could provide higher prediction accuracy. Regarding the performance of FTIR, it should be mentioned that from this instrument a huge amount of data are derived in comparison with e.g. HPLC (18 peaks) enose (12-20 peaks) MSI (18 wavelength) GC/MS (130 peaks). This should be taken into account if data mining should be applied before the implementation of 'MeatReg'.

Supplementary Tables S1\&S2 show that HPLC data is very suitable to predict bacterial counts as the regression methods provide good results in most cases. MSI and GC-MS data are very suitable as well in tandem with kNN-R, SVM-R, RF-R, PLS-R or PC-R. However, GC-MS is not likely to be introduced in the industry due to its high cost, high maintenance and size, compared to more portable MSI platforms such as Videometer.

Aside from the difficulties mentioned before regarding the predictions of certain bacterial counts, electronic nose data is suitable to predict bacterial counts using most methods for modified atmosphere packaging but the appropriate methods for aerobic packaging. For the total bacterial counts under aerobic packaging support vector regression is recommended. For lactic acid bacteria (MRS) all the methods provided accurate predictions while for Enterobacteria (VRBG) SVM-R, linear regression and stepwise linear regression provided the best results. For FT-IR data, aside from pseudomonads and Enterobacteria under aerobic conditions, RF-R provided the best predictions in all cases. Additionally, the results rank PLS-R, kNN-R, PC-R and SVM-R next, which work generally fine for all methods with some exceptions.

\section{Conclusions}

An interactive online platform has been developed to determine the most suitable regression machine learning method that provides the best prediction accuracy of different types of microorganisms involved in beef spoilage using data from five different analytical methods. A thorough approach in selecting the most appropriate strategy for validation of the developed models has been implemented, by providing several methods for calculating the difference between the predicted and the measured values, such as RMSE, the accuracy, the maximum difference between predicted and observed values $\left(\Delta_{\max }\right)$, the bias factor $\left(\mathrm{B}_{f}\right)$ and the accuracy $\left(\mathrm{A}_{f}\right)$ factor. The 
models development, optimisation, and validation is taking place on the server-side via a userfriendly interface, therefore abolishing the need for installing specialised statistical software at the client-side (i.e. the user computer). The analysis output is provided through a comprehensive PDF report which include all optimisation and validation results for each chosen analytical platform and $\mathrm{ML}$ method, as well as all the parameters used during the analysis. As a future extension of the developed platform, we aim to provide a classification ML suite to complement the regression suite presented hereby, to predict spoilage-related discrete values, such as freshness profiles and sensory. Additionally, we aim to extend the compatibility of the data repository to support additional platform commonly applied in food research such as Raman and MALDI-ToF Mass Spectroscopy. Data collected from either FT-IR, image analysis, HPLC or GC-MS combined with appropriate machine learning strategies (for example partial least squares regression, artificial neural networks) could become an interesting tool to monitor food spoilage/freshness through the measurement of biochemical changes occurring in food substrate, without the history of sample to be known (e.g. temperature of storage, the initial contamination, $\mathrm{pH}$ ).

A primary goal of data visualization is to communicate information clearly and efficiently to users via the information graphics selected, such as tables and charts. Effective visualization helps users in analysing and reasoning about data and evidence. It makes complex data more accessible, understandable and usable. Data visualization is both an art and a science.

\section{Authors Contribution}

Lopez developed the "MeatReg" library and the machine learning models described in this work. Ropodi, Pavlidis, Fotopolou, and Gkousari performed the laboratory work, microbiological and instrumental analysis. Peyrodie contributed in developing the sorfML platform and integrated the MeatReg library with the online platform. Panagou managed the data analysis. Nychas designed the experiment and led the wet-lab work. Mohareb managed the implementation the sorfML platform the machine learning work, and led the article preparation together with Nychas. All authors contributed equally to article preparation and approved the final article.

\section{Declaration of interest}

There are no conflicts of interest among authors. All authors have met the criteria for authorship, and have read and approved the final version of the manuscript. This work is not under consideration elsewhere. 


\section{References}

Argyri, A., A., Panagou, Z., E., Tarantilis, A., P., . . E., G.-J. (2010). Rapid qualitative and quantitative detection of beef fillets spoilage based on Fourier transform infrared spectroscopy data and artificial neural networks. 145(1), 9.

Argyri, A. A., Jarvis, R. M., Wedge, D., Xu, Y., Panagou, E. Z., Goodacre, R., \& Nychas, G.-J. E. (2013). A comparison of Raman and FT-IR spectroscopy for the prediction of meat spoilage. Food Control, 29(2), 461-470. doi: http://dx.doi.org/10.1016/i.foodcont.2012.05.040

Argyri, A. A., Mallouchos, A., Panagou, E. Z., \& Nychas, G. J. (2015). The dynamics of the HS/SPME$\mathrm{GC} / \mathrm{MS}$ as a tool to assess the spoilage of minced beef stored under different packaging and temperature conditions. Int J Food Microbiol, 193, 51-58. doi: 10.1016/j.ijfoodmicro.2014.09.020

Baietto, M., Wilson, A. D., Bassi, D., \& Ferrini, F. (2010). Evaluation of three electronic noses for detecting incipient wood decay. Sensors (Basel), 10(2), 1062-1092. doi: 10.3390/s100201062

Baranyi, J., Pin, C., \& Ross, T. (1999). Validating and comparing predictive models. Int J Food Microbiol, 48(3), 159-166.

Baranyi, J., \& Roberts, T. A. (1994). A dynamic approach to predicting bacterial growth in food. Int J Food Microbiol, 23(3-4), 277-294.

Beygelzimer, A., Kakadet, S., Langford, J., Arya, S., \& Mount, D. (2013). FNN: Fast Nearest Neighbor Search Algorithms and Applications. (Version 1.1) [R package]. https://cran.rproject.org/package=FNN.

Boser, B. E., Guyon, I. M., \& Vapnik, V. N. (1992). A Training Algorithm for Optimal Margin Classifiers \%U http://doi.acm.org/10.1145/130385.130401. In D. Haussler (Ed.), Proceedings of the 5th Annual Workshop on Computational Learning Theory (COLT'92) (pp. 144--152). Pittsburgh, PA, USA: ACM Press.

Breiman, L. (2001). Random Forests. Mach. Learn., 45(1), 5-32. doi: 10.1023/a:1010933404324

Commission regulation (EC) No 2073/2005 on microbiological criteria for foodstuffs, OJ L C.F.R. (2005).

Daugaard, S. B., Adler-Nissen, J., \& Carstensen, J. M. (2010). New vision technology for multidimensional quality monitoring of continuous frying of meat. Food Control, 21(5), 626632. doi: https://doi.org/10.1016/i.foodcont.2009.09.007

Dimitriadou, E., Hornik, K., Leisch, F., Meyer, D., \& Weingessel, A. (2011). e1071: Misc Functions of the Department of Statistics (e1071), TU Wien.

Doulgeraki, A. I., Ercolini, D., Villani, F., \& Nychas, G. J. (2012). Spoilage microbiota associated to the storage of raw meat in different conditions. Int J Food Microbiol, 157(2), 130-141. doi: 10.1016/j.ijfoodmicro.2012.05.020

Friedman, J., Hastie, T., \& Tibshirani, R. (2010). Regularization Paths for Generalized Linear Models via Coordinate Descent. J Stat Softw, 33(1), 1-22.

Höskuldsson, A. (1988). PLS regression methods. Journal of Chemometrics, 2(3), 211-228. doi: 10.1002/cem.1180020306

Kendall, M. G. (1957). A Course in Multivariate Analysis: Griffin.

Koutsoumanis, K. (2009). Modeling food spoilage in microbial risk assessment. J Food Prot, 72(2), 425-427.

Liaw, A., \& Wiener, M. (2002). Classification and Regression by randomForest. $R$ News, 2(3), 18-22. doi: citeulike-article-id:1121494

Mataragas, M., Skandamis, P., Nychas, G. J., \& Drosinos, E. H. (2007). Modeling and predicting 
spoilage of cooked, cured meat products by multivariate analysis. Meat Sci, 77(3), 348-356. doi: 10.1016/j.meatsci.2007.03.023

Mevik, B.-H., \& Wehrens, R. (2007). The pls Package: Principal Component and Partial Least Squares Regression in R. Journal of Statistical Software, 18(2), 1-24.

Mohareb, F., Iriondo, M., Doulgeraki, A. I., Van Hoek, A., Aarts, H., Cauchi, M., \& Nychas, G.-J. E. (2015). Identification of meat spoilage gene biomarkers in Pseudomonas putida using gene profiling. Food Control, 57, 152-160. doi: http://dx.doi.org/10.1016/i.foodcont.2015.04.007

Mohareb, F., Papadopoulou, O., Panagou, E., Nychas, G.-J., \& Bessant, C. (2016). Ensemble-based support vector machine classifiers as an efficient tool for quality assessment of beef fillets from electronic nose data. [10.1039/C6AY00147E]. Analytical Methods, 8(18), 3711-3721. doi: 10.1039/C6AY00147E

Nychas, G.-J. E., Marshall, D., \& Sofos, J. (2007). Chapter 6; Meat Poultry and Seafood. Eds Doyle: Beuchat and Montville ASM Press.

Nychas, G.-J. E., Panagou, E. Z., \& Mohareb, F. (2016). Novel approaches for food safety management and communication. Current Opinion in Food Science, 12, 13-20. doi: http://dx.doi.org/10.1016/i.cofs.2016.06.005

Nychas, G. J., Skandamis, P. N., Tassou, C. C., \& Koutsoumanis, K. P. (2008). Meat spoilage during distribution. Meat Sci, 78(1-2), 77-89. doi: 10.1016/j.meatsci.2007.06.020

Nychas, G. J. E., Skandamis, P. N., Tassou, C. C., \& Koutsoumanis, K. P. (2008). Meat spoilage during distribution. Meat Sci, 78(1-2), 77-89. doi: Doi 10.1016/J.Meatsei.2007.06.020

Panagou, E. Z., Mohareb, F. R., Argyri, A. A., Bessant, C. M., \& Nychas, G. J. (2011). A comparison of artificial neural networks and partial least squares modelling for the rapid detection of the microbial spoilage of beef fillets based on Fourier transform infrared spectral fingerprints. Food Microbiol, 28(4), 782-790. doi: 10.1016/j.fm.2010.05.014

Panagou, E. Z., Papadopoulou, O., Carstensen, J. M., \& Nychas, G. J. (2014). Potential of multispectral imaging technology for rapid and non-destructive determination of the microbiological quality of beef filets during aerobic storage. Int J Food Microbiol, 174, 1-11. doi: 10.1016/j.ijfoodmicro.2013.12.026

Rajamäki, T., Alakomi, H.-L., Ritvanen, T., Skyttä, E., Smolander, M., \& Ahvenainen, R. (2006). Application of an electronic nose for quality assessment of modified atmosphere packaged poultry meat. Food Control, 17(1), 5-13. doi: http://dx.doi.org/10.1016/i.foodcont.2004.08.002

Ropodi, A. I., Panagou, E. Z., \& Nychas, G. J. E. (2016). Data mining derived from food analyses using non-invasive/non-destructive analytical techniques; determination of food authenticity, quality \&amp; safety in tandem with computer science disciplines. Trends in Food Science \& Technology, 50, 11-25. doi: http://dx.doi.org/10.1016/j.tifs.2016.01.011

Ropodi, A. I., Pavlidis, D. E., Mohareb, F., Panagou, E. Z., \& Nychas, G. J. E. (2015). Multispectral image analysis approach to detect adulteration of beef and pork in raw meats. Food Research International, 67, 12-18. doi: https://doi.org/10.1016/i.foodres.2014.10.032

Silverman, B. W., \& Jones, M. C. (1989). E. Fix and J.L. Hodges(1951): an important contribution to nonparametric discriminant analysis and density estimation. International Statistical Review, 57(3), 233-247.

Skandamis, P. N., \& Nychas, G. J. (2001). Effect of oregano essential oil on microbiological and physico-chemical attributes of minced meat stored in air and modified atmospheres. J Appl Microbiol, 91(6), 1011-1022.

Van Wezemael, L., Verbeke, W., de Barcellos, M. D., Scholderer, J., \& Perez-Cueto, F. (2010). Consumer perceptions of beef healthiness: results from a qualitative study in four European 
countries. BMC Public Health, 10, 342. doi: 10.1186/1471-2458-10-342

Wang, D., Wang, X., Liu, T., \& Liu, Y. (2012). Prediction of total viable counts on chilled pork using an electronic nose combined with support vector machine. Meat Science, 90(2), 373-377. doi: http://dx.doi.org/10.1016/i.meatsci.2011.07.025

Wilkinson, G. N., \& Rogers, C. E. (1973). Symbolic descriptions of factorial models for analysis of variance. Applied Statistics, 22, 392-399.

$\mathrm{Xu}$, Q.-S., \& Liang, Y.-Z. (2001). Monte Carlo cross validation. Chemometrics and Intelligent Laboratory Systems, 56(1), 1-11. doi: http://dx.doi.org/10.1016/S0169-7439(00)00122-2

Xu, Q.-S., Liang, Y.-Z., \& Du, Y.-P. (2004). Monte Carlo cross-validation for selecting a model and estimating the prediction error in multivariate calibration. Chemometrics, 18, 112-120. 
Table 1.: Kinetic parameters (following data fitting, calculated with the model of Baranyi and Robert 1994) of the members of microbial association of minced meat [Total Viable Counts; initial counts $5.48 \pm 0.2$ log cfu, pseudomonads spp.] stored under aerobic conditions at 4 and $10^{\circ} \mathrm{C}$, as function of the storage temperature and the packaging type.

\begin{tabular}{|c|c|c|c|c|c|c|c|}
\hline & Microbial group & $\mu_{\max }{ }^{a}$ & $\begin{array}{l}\text { Lag } \\
\text { phase } \\
\text { (h) }\end{array}$ & $\begin{array}{l}\text { y0 (log } \\
\text { cfu/g) }\end{array}$ & $\begin{array}{l}\text { yEnd } \\
\text { (log } \\
\text { cfu/g) }\end{array}$ & $\begin{array}{c}\text { Standard } \\
\text { error of } \\
\text { fitting }\end{array}$ & $\begin{array}{c}\text { Adjusted } R^{2} \text {, } \\
\text { statistics of } \\
\text { the fitting }\end{array}$ \\
\hline \multirow[t]{6}{*}{$4^{\circ} \mathrm{C}$} & Total Viable count & 0.057 & 35.78 & 5.60 & 9.38 & 0.180 & 0.988 \\
\hline & Pseudomonads & 0.080 & 18.93 & 4.12 & 9.32 & 0.200 & 0.990 \\
\hline & B. thermosphacta & 0.075 & 21.03 & 4.24 & 7.82 & 0.160 & 0.986 \\
\hline & Lactic acid bacteria & 0.036 & 64.73 & 4.30 & 6.35 & 0.205 & 0.907 \\
\hline & Enterobacteriaceae & 0.041 & 45.78 & 1.75 & nd & 0.203 & 0.967 \\
\hline & Yeasts-molds & 0.025 & 17.58 & 4.70 & 6.67 & 0.159 & 0.943 \\
\hline \multirow[t]{6}{*}{$10^{\circ} \mathrm{C}$} & Total Viable count & 0.126 & nd & 5.50 & 10.90 & 0.218 & 0.964 \\
\hline & Pseudomonads & 0.124 & nd & 5.72 & 10.78 & 0.300 & 0.931 \\
\hline & B. thermosphacta & 0.143 & nd & 4.36 & 7.73 & 0.237 & 0.958 \\
\hline & Lactic acid bacteria & 0.126 & nd & 3.40 & 7.15 & 0.200 & 0.957 \\
\hline & Enterobacteriaceae & 0.053 & nd & 0.85 & nd & 0.463 & 0.658 \\
\hline & Yeasts-molds & 0.103 & nd & 3.82 & 5.7 & 0.306 & 0.806 \\
\hline
\end{tabular}

${ }^{a}$ Maximum specific growth rate $h \_1$. 
Table 2.: Kinetic parameters (following data fitting, calculated with the model of Baranyi and Robert 1994) of the members of microbial association of minced meat [Total Viable Counts; initial counts $5.48 \pm 0.2 \mathrm{log} \mathrm{cfu}$, pseudomonads] stored under modified atmosphere conditions at 4 and $10^{\circ} \mathrm{C}$, as function of the storage temperature and the packaging type

\begin{tabular}{|c|c|c|c|c|c|c|c|c|}
\hline & Microbial group & $\mu_{\max }{ }^{a}$ & $\begin{array}{c}\text { Lag } \\
\text { phase } \\
\text { (h) }\end{array}$ & $\begin{array}{l}\text { y0 (log } \\
\text { cfu/g) }\end{array}$ & $\begin{array}{l}\text { yEn } \\
\text { (lo } \\
\text { cfu) }\end{array}$ & & & $\begin{array}{l}\text { Adjusted } R^{2} \text {, } \\
\text { statistics of } \\
\text { the fitting }\end{array}$ \\
\hline & \multicolumn{2}{|l|}{ Microbial group } & $\mu_{\max }$ & lag & yo & yEnd & Se(fit) & $\mathbf{R}^{\wedge} \mathbf{2}$ _stat \\
\hline \multirow[t]{6}{*}{$4^{\circ} \mathrm{C}$} & Total Viable count & & 0.040 & 74.23 & 5.86 & 7.81 & 0.166 & 0.963 \\
\hline & Pseudomonads & & 0.050 & 60.75 & 4.09 & 7.35 & 0.287 & 0.917 \\
\hline & B. thermosphacta & & 0.070 & 36.35 & 4.51 & 7.78 & 0.227 & 0.965 \\
\hline & Lactic acid bacteria & & 0.021 & 62.29 & 4.49 & 6.05 & 0.105 & 0.972 \\
\hline & Enterobacteriaceae & & 0.052 & 112.35 & 2.01 & 3.29 & 0.144 & 0.944 \\
\hline & Yeasts-molds & & 0.024 & 25.60 & 4.83 & 6.07 & 0.155 & 0.871 \\
\hline \multirow[t]{6}{*}{$10^{\circ} \mathrm{C}$} & Total Viable count & & 0.116 & nd & 5.41 & 7.99 & 0.223 & 0.935 \\
\hline & Pseudomonads & & 0.075 & nd & 5.40 & 7.10 & 0.420 & 0.892 \\
\hline & B. thermosphacta & & 0.100 & nd & 4.20 & 7.06 & 0.247 & 0.949 \\
\hline & Lactic acid bacteria & & 0.094 & nd & 3.29 & 6.35 & 0.177 & 0.979 \\
\hline & Enterobacteriaceae & & 0.019 & nd & 0.25 & nd & 0.326 & 0.419 \\
\hline & Yeasts-molds & & 0.042 & nd & 3.87 & nd & 0.654 & 0.796 \\
\hline
\end{tabular}




\section{List of Figures}

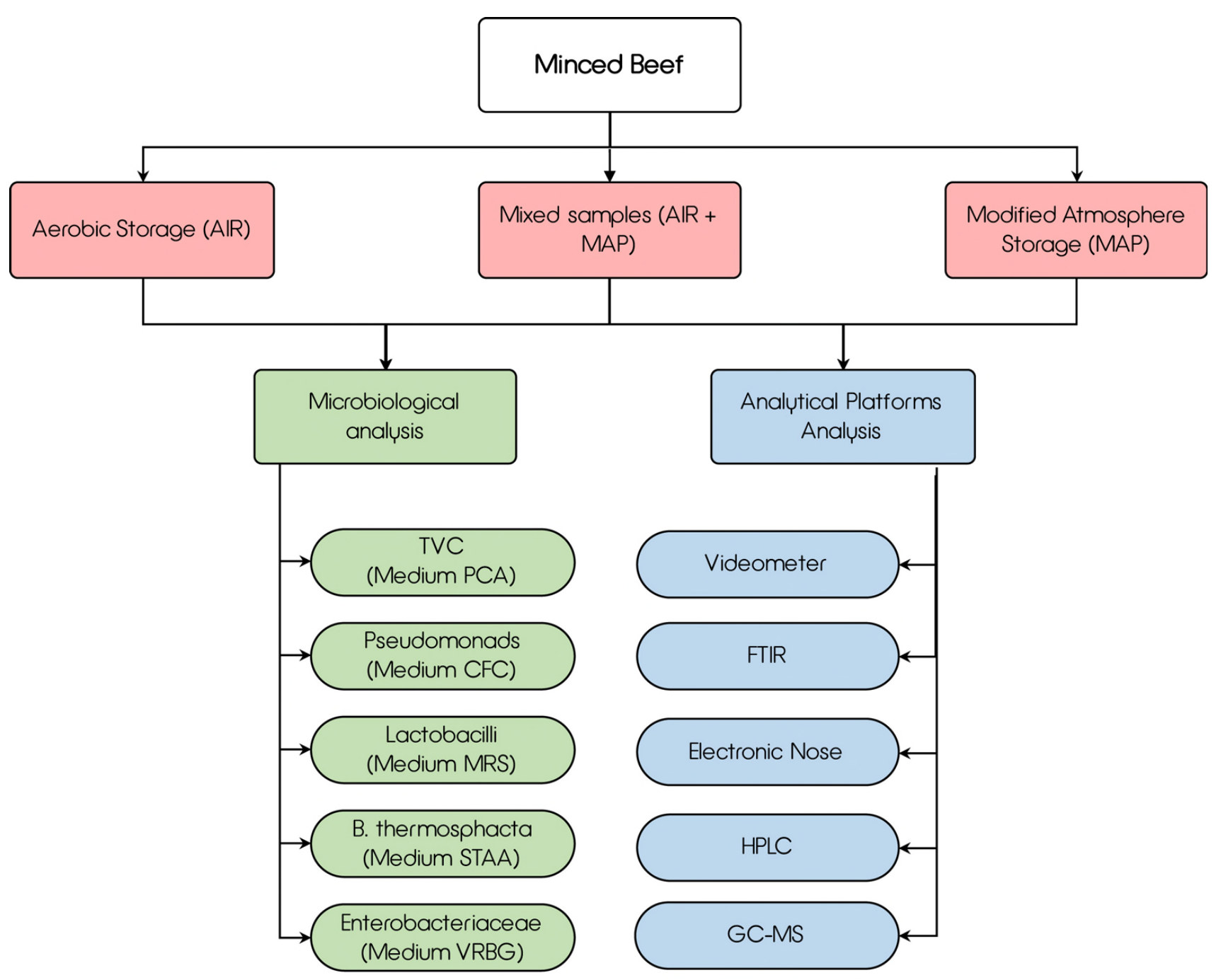

Figure 1. Minced beef is stored using three different packaging conditions (highlighted in red). The samples stored under both packaging conditions go through microbiological analysis (highlighted in green) and analysis using metabolomics platforms (highlighted in blue). 


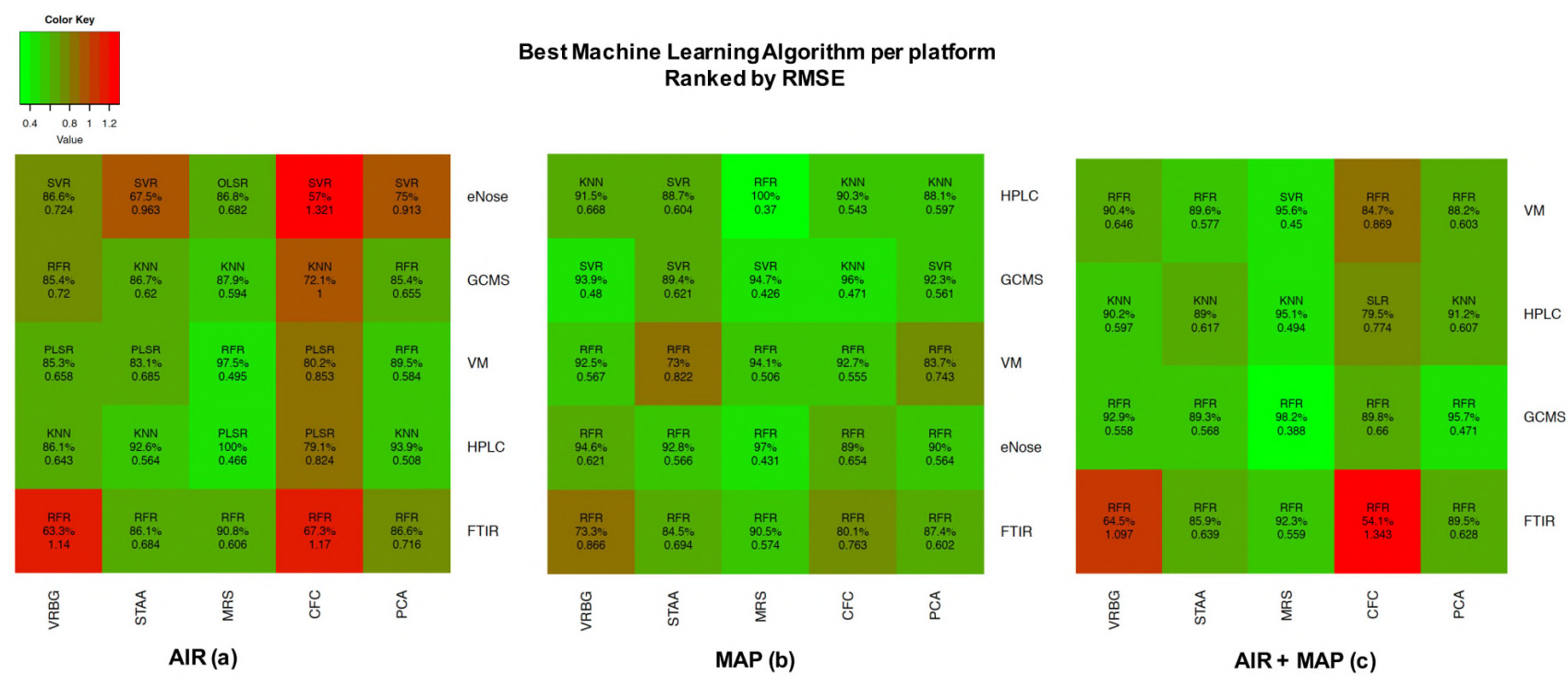

Figure 2. Suitability of analytical platforms to predict the bacterial count for beef samples stored under: aaerobic packaging (AIR); b - modified atmosphere packaging (MAP); and c- Mixed packaging (AIR +MAP). Green indicates a low RMSE while red indicates a higher RMSE. 


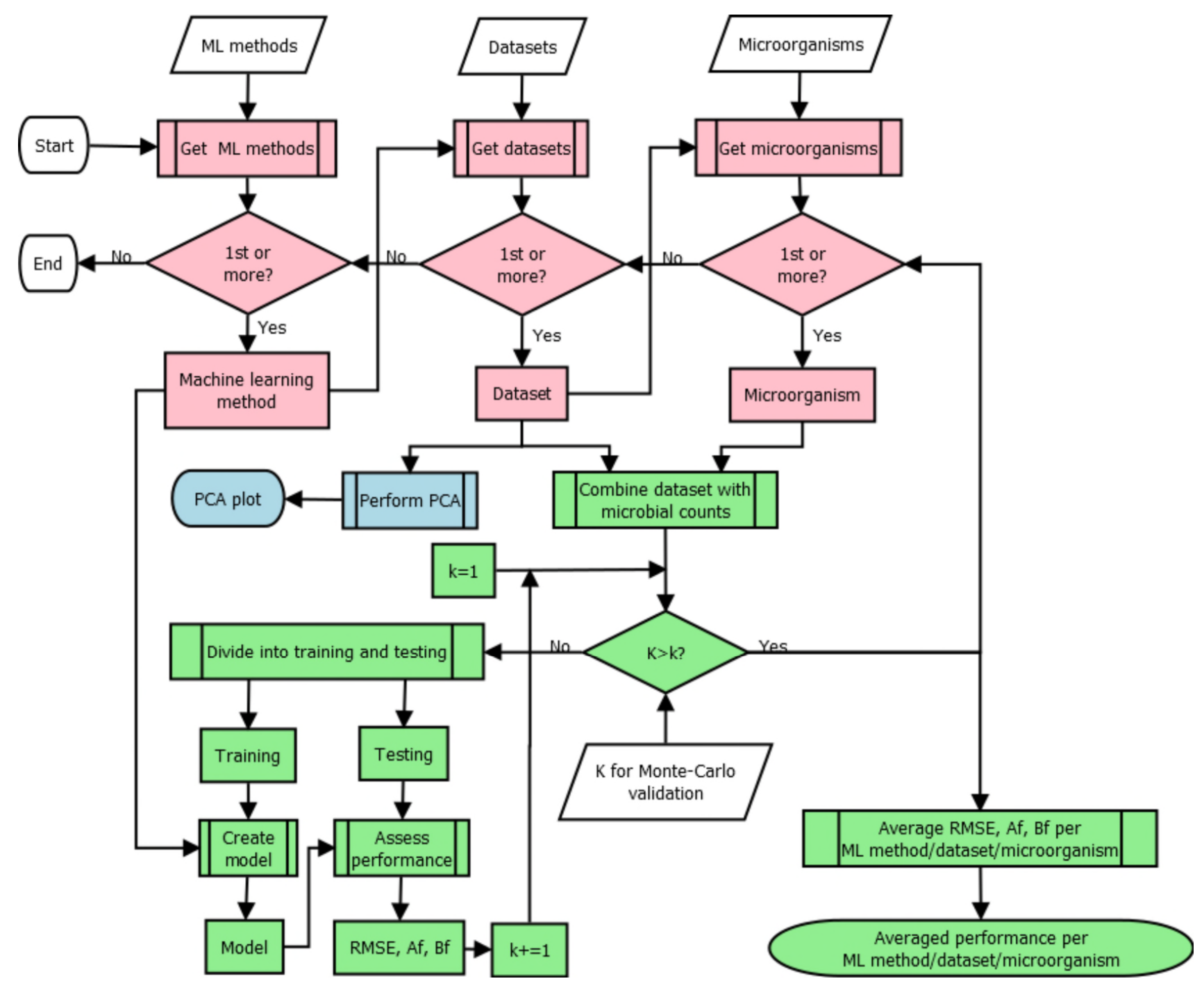

Figure 3. Simplified workflow of the analysis. The selection of machine learning method, dataset and microorganisms is highlighted in red; the production of PCA plots is highlighted in blue and the creation of statistical models and assessment of their performance is highlighted in green. 


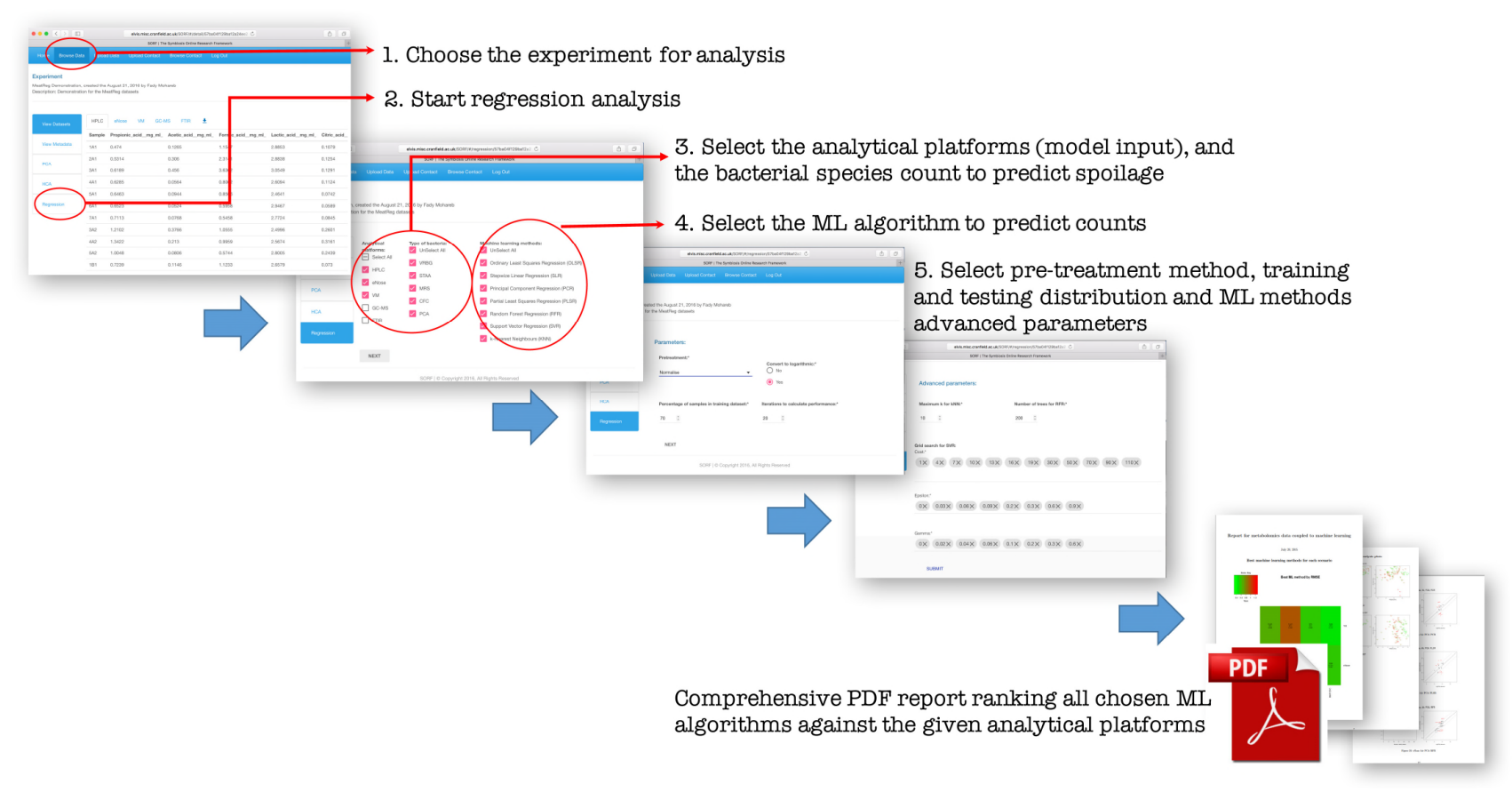

Figure 4. Screenshots of the sorfML web platform form showing steps involved in performing online regression analysis. 